\title{
Arc Second Alignment of International X-Ray Observatory Mirror Segments in a Fixed Structure
}

\author{
Tyler C. Evans \\ 301-286-2677, tyler.evans@nasa.gov \\ Kai-Wing Chan \\ 301-286-6238, Kai-Wing.Chan-1@nasa.gov \\ NASA Goddard Space Flight Center \\ Greenbelt, MD 20771
}

\begin{abstract}
The optics for the International X-Ray Observatory (IXO) require alignment and integration of about fourteen thousand thin mirror segments to achieve the mission goal of 3.0 square meters of effective area at 1.25 $\mathrm{keV}$ with an angular resolution of five arc seconds. These mirror segments are $0.4 \mathrm{~mm}$ thick, and 200 to $400 \mathrm{~mm}$ in size, which makes it hard not to impart distortion at the subarc second level. This paper outlines the precise alignment, verification testing, and permanent bonding techniques developed at NASA's Goddard Space Flight Center (GSFC). These techniques are used to overcome the challenge of transferring thin mirror segments from a temporary mount to a fixed structure with are second alignment and minimal figure distortion. Recent advances in technology development in addition to the automation of several processes have produced significant results. This paper will highlight the recent advances in alignment, testing, and permanent bonding techniques as well as the results they have produced. ${ }^{12}$
\end{abstract}

\section{TABLE OF CONTENTS}

\begin{tabular}{l} 
1. INTRODUCTION............ \\
\hline 2. MIRROR SEGMENTS \\
\hline 3. TEMPORARY MOUNT \\
\hline 4. MIRROR ALIGNMENT \\
\hline 5. VERIFICATION TESTING
\end{tabular}

\section{INTRODUCTION}

Aligning thin glass segments used for the optics of the International X-Ray Observatory (IXO) poses an interesting challenge. IXO is a pre-Phase A project designed at building upon the success of previous X-ray missions such as Chandra and XMM Newton. (For an IXO mission background see [1]). It will have a much larger effective area than any previous x-ray mission of 3.0 square meters at $1.25 \mathrm{keV}$ with an angular resolution of five arc seconds. A Wolter-I type telescope design was selected to enable the

\footnotetext{
${ }^{1}$ U.S. Government work not protected by U.S. copyright

${ }^{2}$ IEEEAC paper $4 X X X X$, Version X, Updated October 27, 2009.
}

mirror segments to be nested in order to increase the effective area. In the Wolter-I type design, the incoming $x$ ray photons graze off of a primary mirror and a secondary mirror at a very small angle to get to the detector. The nested mirror segments were selected to be $0.4 \mathrm{~mm}$ thin to conserve mass and maximize collecting area. Meeting the angular resolution requirement of five arc seconds with such thin glass segments presents a challenge.

To accommodate all of the mirrors for the telescope, a modular design was conceived. The Flight Mirror Assembly (FMA) will support 60 modules arranged in three rings, 12 inner, 24 middle, and 24 outer [2]. There will be 200 to 280 mirror segments per module for a total of about fourteen thousand mirror segments. The primary and secondary mirrors must be aligned to each other to meet the strict angular resolution requirement. In addition, all of the mirror pairs must focus to essentially the same point. Because of the thin nature of the mirror segments being used, they are easily distorted.

There are currently three approaches being developed to solve the challenge of aligning and mounting the mirror segments into a permanent structure. In the first approach, the mirror is adjusted with small high resolution linear actuators to correct for axial and figure errors. This method is being pursued by a team at the Smithsonian Astrophysical Observatory (SAO)[3]. The second method involves forcing the mirror segment into a prescribed geometry. This approach is being investigated at the European Space Agency (ESA) and associated industries [4]. The third method is to preserve the fabricated state of the mirror and not introduce any distortion or figure error throughout the alignment and mounting processes. This third method is being developed at NASA's Goddard Space Flight Center (GSFC) and will be discussed in this paper.

\section{MirRor SEgMents}

\section{Mirror Segment Background}

In order to meet the large effective area requirement and minimize weight, about fourteen thousand glass mirror segments that are $0.4 \mathrm{~mm}$ thick are used in a nested shell pattern. The mirror segments are slumped from D263 glass onto polished mandrels [5]. The mirror segments are $200 \mathrm{~mm}$ long in the axial direction and have a 
circumferential span of up to $360 \mathrm{~mm}$. This makes each mirror about the size of a standard sheet of paper.

The mirrors are grouped into three rings of modules with 12 to 24 modules in each ring, with an average of 240 mirror segments in each module. The combined group of mirror segments, modules, and support structure is called the Flight Mirror Assembly (FMA). The total mass of the FMA is about $1300 \mathrm{~kg}[2]$.

Table 1. X-Ray mission angular resolution and mass to area comparisons

\begin{tabular}{|c|c|c|c|}
\hline Mission & CEANDRA & WOANeston & mo \\
\hline $\begin{array}{l}\text { Angular } \\
\text { Resolution } \\
\text { (arcsec) }\end{array}$ & 0.5 & 14 & 5 \\
\hline $\begin{array}{c}\text { Mass per } \\
\text { square meter } \\
\left(\mathrm{Kg} / \mathbf{m}^{2}\right)\end{array}$ & 18300 & 2300 & 270 \\
\hline
\end{tabular}

Table 1 helps give a reference from optics of previous $\mathrm{x}$-ray missions. IXO has a much lower mass to area ratio than previous $\mathrm{x}$-ray missions. This is accomplished by using many thin mirror segments to maximize the collecting area while minimizing the weight. This comes at a trade-off however, as the thin nature of the segments equals a low stiffness which makes them easy to deform or distort.

\section{Preliminary Budget of Error Contributions}

As shown in Error! Not a valid bookmark selfreference.Table 2, a total of 1.26 arcsec of error is budgeted to be introduced to the mirror during the temporary mount and permanent bond procedures. This can be further broken down into 0.89 arcsec for the temporary mount and 0.89 arcsec for the permanent bond. This feeds into the plan to have a final error of 4.14 arcsec at the FMA stage. (Note that to calculate the addition of errors, the square root is taken of the sum of the squares.)
Table 2. Error budget from fabrication to flight

\begin{tabular}{|c|c|c|c|}
\hline & $\begin{array}{c}\text { Running } \\
\text { Sum } \\
\text { (arcsec) }\end{array}$ & $\begin{array}{l}\text { Individual } \\
\text { Process } \\
\text { Contribution } \\
\text { (arcsec) }\end{array}$ \\
\hline \multirow{3}{*}{ 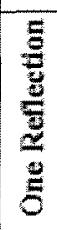 } & Forming Mandrel & 1.47 & 1.47 \\
\hline & $\begin{array}{c}\text { Mirror } \\
\text { Fabrication }\end{array}$ & 2.41 & 1.91 \\
\hline & $\begin{array}{c}\text { Temporary } \\
\text { Mount and } \\
\text { Permanent Bond }\end{array}$ & 2.72 & 1.26 \\
\hline \multirow{2}{*}{ 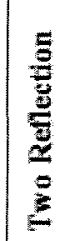 } & Module & 3.86 & 0.34 \\
\hline & FMA & 4.14 & 1.50 \\
\hline
\end{tabular}

The value of 0.89 arcsec for the permanent bond procedure is further broken down in Figure 1. These values are measured using interferomic metrology, Hartmann tests, and some are not yet able to be measured. The Hartmann test mainly focuses on cone angle variation, which is the largest contributor at this time.

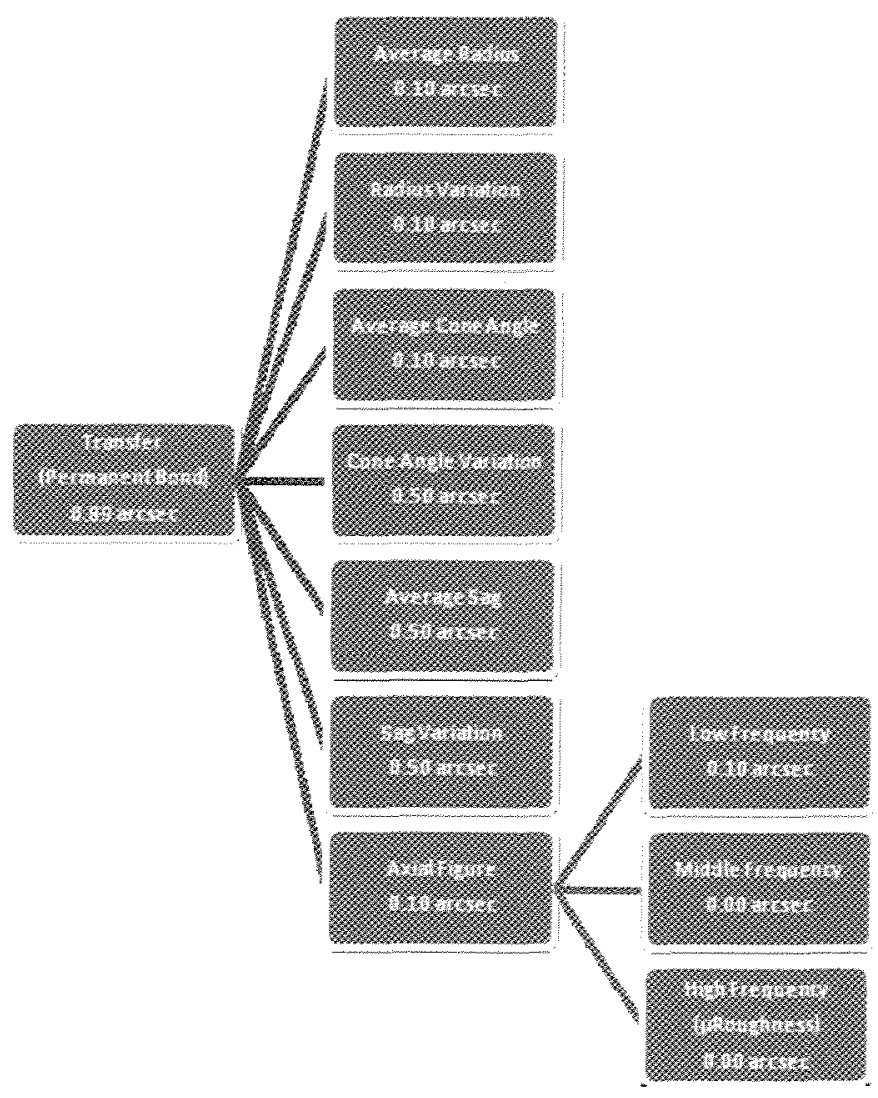

Figure 1 - Error budget for permanent bond (values are preliminary and may change)

The radius variation, cone angle variation, average sag, and 


\section{National Aeronautics and}

Space Administration

sag variation are all measured with an interferometer and

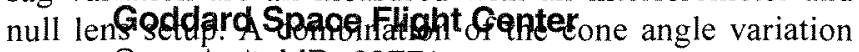
and rad

\section{TEMPorary Mount}

The temporary mount method being used at NASA's Goddard Space Flight Center (GSFC) is the suspension Reply to Attmount [6]. The idea behind the suspension mount is to preserve the optical figure of the mirror during alignment and bonding into a permanent structure. First, the mirror is hung using four strings to minimize the gravity distortion on the mirror as shown in Figure 2.

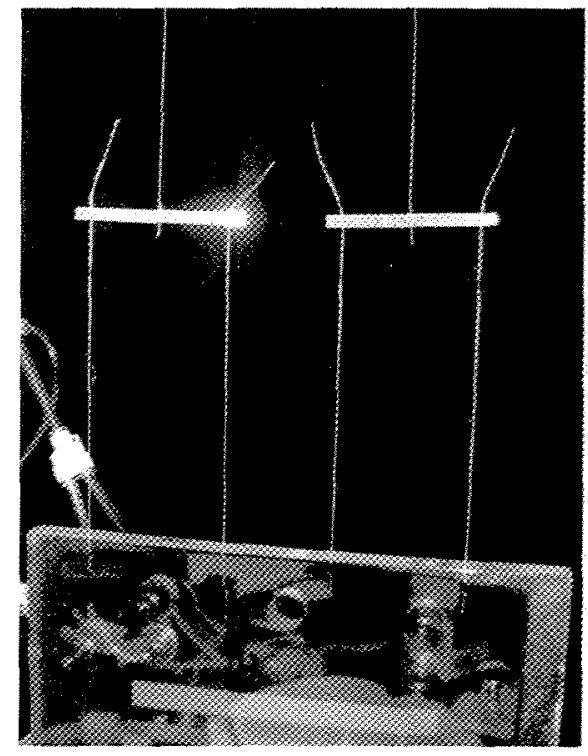

Figure 2 - Four string suspension mount

Once the mirror is hanging vertically, it is captured by temporary mount hardware called a strongback. The strongback has a set of pins protruding from its front surface. These pins are set in near-frictionless air bearings so that they apply minimal force when making contact with the mirror. The pins are bonded to the back of the mirror as shown in Figure 3, but are still able to float freely to compensate for the mirror swaying or moving. When the mirror settles into its relaxed state, the back of the pins are bonded to strongback, to freeze them in place. This essentially freezes the mirror in its hanging state where the distortion is minimized.

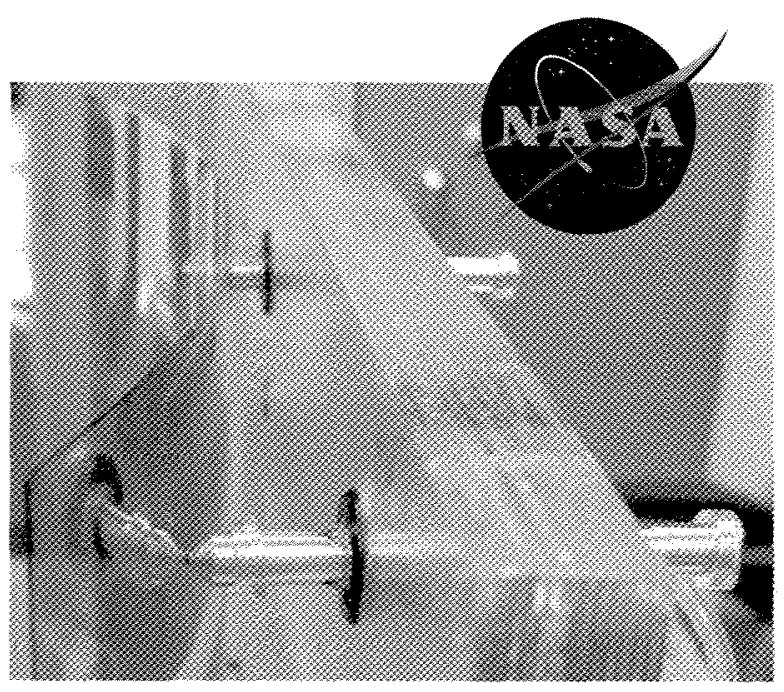

Figure 3 - Pins in air bearings bonded to mirror

Once the mirror is on the strongback, it can be transported and tilted into alignment.

\section{Mirror Alignment}

Each mirror segment is aligned using a six degree of freedom hexapod. The hexapod has a repeatability of \pm 0.5 $\mu \mathrm{m}$ in the linear $\mathrm{X}, \mathrm{Y}$, and $\mathrm{Z}$ directions. The controller outputs the absolute position of the hexapod in $X, Y, Z$ coordinates in $\mathrm{mm}$ to four decimal places. The rotational position of the hexapod in $\mathrm{U}, \mathrm{V}$, and $\mathrm{W}$ coordinates is reported in degrees to four decimal places. Knowing the absolute position of the mirror to this level of accuracy enables calculations to be performed to determine the necessary adjustments for optimizing the image.

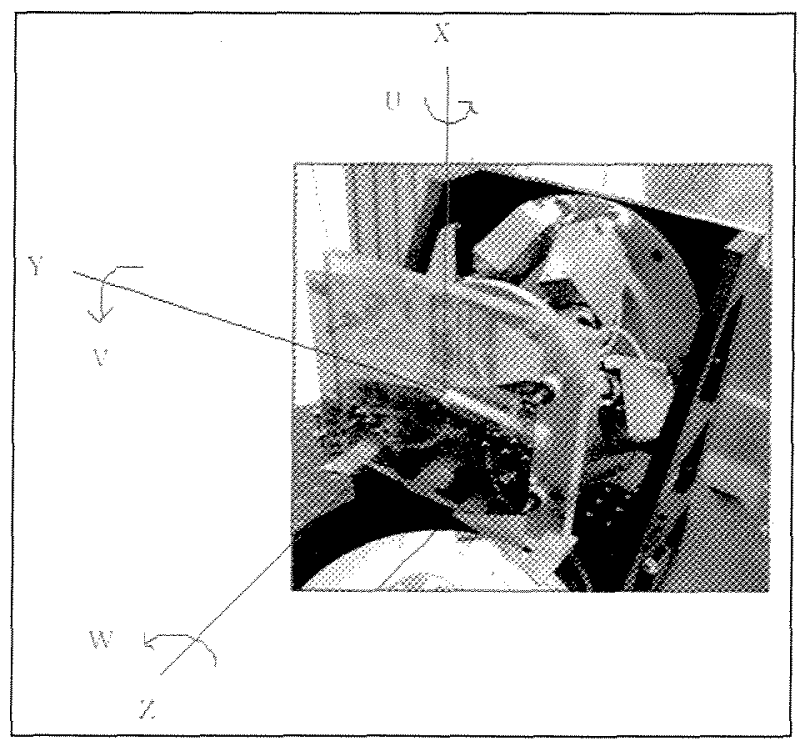

Figure 4 - Hexapod coordinate system

The alignment is mainly adjusted by titling the mirror in the $\mathrm{V}$ direction, and by titling the mirror in the $\mathrm{W}$ direction. The final way to obtain a better image is to adjust the focal distance by moving the CCD camera at the end of the beam. 
There are three main focal distances that are used for the specific mirror segment being tested right now. Different sized mirror segments will have different individual focal distances, but the combined focal distance of 8.4 meters will remain the same.

Table 3. Focal distances of various 485 type segments

\begin{tabular}{|c|c|}
\hline Type of Segment & Focal Distance (m) \\
\hline Primary & 17.056 \\
\hline Secondary & 5.654 \\
\hline Primary and Secondary & 8.400 \\
\hline
\end{tabular}

To achieve this long of a focal distance when the mirror is in a vertical position, a light source is positioned above the mirrors, shone downwards, and then bent 90 degrees so that it is parallel with the optical bench surface. It is then bounced back and forth using fold mirrors to achieve the necessary focal length. The light source is a red beam assumed to have a wavelength of $633 \mathrm{~nm}$, which is in the visible light spectrum.

The mirror reflection focuses smaller and smaller until the arc focuses to a small hourglass shape as shown in Figure 5. Past the focus, the arc becomes inverted, and grows in size. Where this focus is located determines part of the alignment. The shape of the hourglass itself determines the rest of the alignment. The shape of the hourglass is determined by performing a Hartmann test.

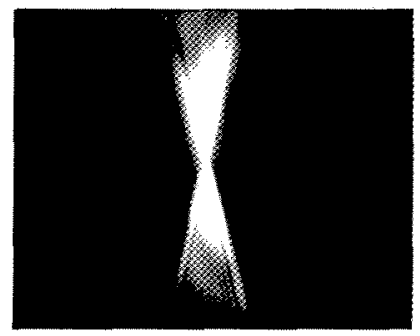

Figure 5-Image of mirror reflection at focus

Due to the light source generating a beam of light with a wavelength of about $633 \mathrm{~nm}$, there is a noticeable diffraction effect in the image. The longer the focal length, the larger the diffraction, therefore the primary segment poses challenges in alignment due to its seventeen meter focal length.

In order to achieve a good result, the mirror must be titled at a very specific angle in which the light distribution at the focus must be symmetrically distributed across the hourglass shape. A rough estimate of this symmetrical distribution of light can be done by simply looking at the image and correcting. Fine tune adjustments are calculated using the analyzed data. Once a Hartmann test is complete, the general shape of the data set in addition to the magnitude of the errors can be used in conjunction with a set of equations to calculate the necessary adjustments needed for the optimal result. Because the relative position of the mirror between tests is known from the hexapod coordinates, it is possible to quantitatively calculate adjustments. Once a mirror is set-up, the automation of the Hartmann test and data analysis on-site makes it possible to run a test and have results in five minutes. This allows for multiple adjustments to be made and to run iterations to perfect the alignment of the mirror segment.

\section{Verification TESTING}

\section{Test Fundamentals}

A modified Hartmann test is used to test the alignment of the mirror. To perform the test, a mask is used to cover the reflection light coming off of the mirror (see Figure 6). Only a specific slit of light is allowed to pass through the mask. The mask is then rotated to allow light from different strips of the mirror to be analyzed independently.

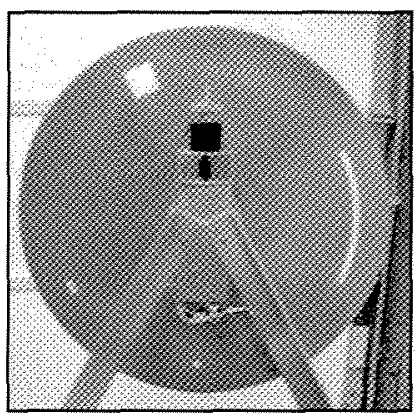

Figure 6-Hartmann mask

In regards to the hourglass shaped focused image, when only a thin segment of the reflection arc is allowed to pass through the Hartmann mask, a line is displayed. When the lines formed by each stripe of the mirror are put together, they form the hourglass shape as shown in Figure 7.

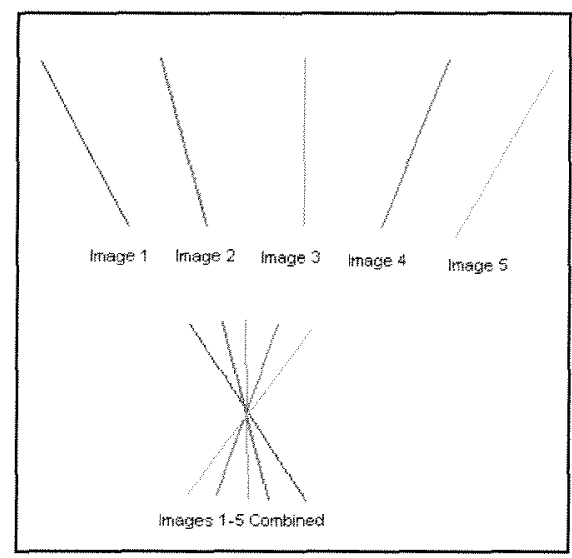

Figure 7 -Combined image explanation (only five images shown to simplify diagram) 
A CCD camera is used to capture an image of each line recording the brightness value of each pixel. The theoretical centroid of the brightness values should be in the center of the hourglass. Therefore the alignment error can be determined from the deviation between the centroids of each of the separate images.

The final outcome of the test is a plot showing the deviation of each centroid location from the average location as shown in Figure 8. Motorized linear stages and rotational motor have been utilized to automate this entire test.

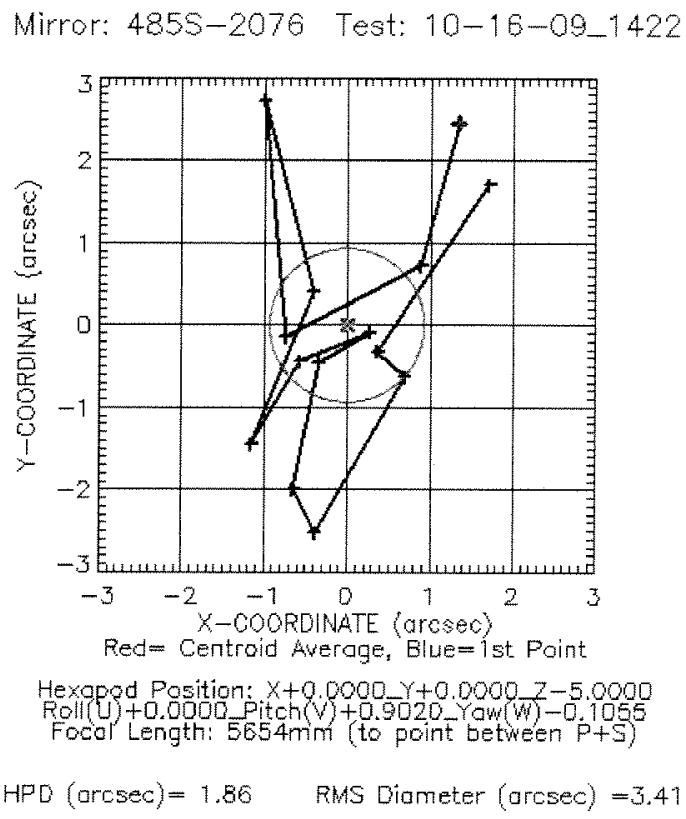

Figure 8-Sample plot of centroids

The mirror segment alignment parameters are labeled on the graph to track settings used to achieve the image. This helps to understand what changed between trials to improve or degrade the image. The parameters are listed in five major categories. The mirror number is reported to show which mirror is being used. The test number reports the date and time ( 24 hour format) that the test was performed. The hexapod position shows the coordinates that the hexapod was programmed to in order to translate and tilt the mirror to the alignment used during the test. The focal length reports the distance between a fixed point PS and the CCD camera. The point PS is a point located $24 \mathrm{~mm}$ above the top of the secondary mirror or $26 \mathrm{~mm}$ below the bottom of the primary mirror in the permanently mounted configuration. The HPD and RMS ratings give a value of the spread of the centroids which is used to rate the mirror. The HPD rating of the mirror stands for "half power diameter". It is the diameter of the circle around the average centroid that would contain half of the points. It is signified by the magenta circle in Figure 8. The blue cross signifies the first data point taken, which helps illustrate the shape of the mirror by tracking the individual points with the order they were taken in. The red $x$ indicates the average of all the centroids.

\section{Data Analysis}

The data that is output after the Hartmann test is a set of images of single lines that when combine would form the "hourglass shape" shown in Figure 7. Each image is analyzed independently to find the angle of a line that passes through the sliver of light. This line is represented by a dashed line in Figure 9.

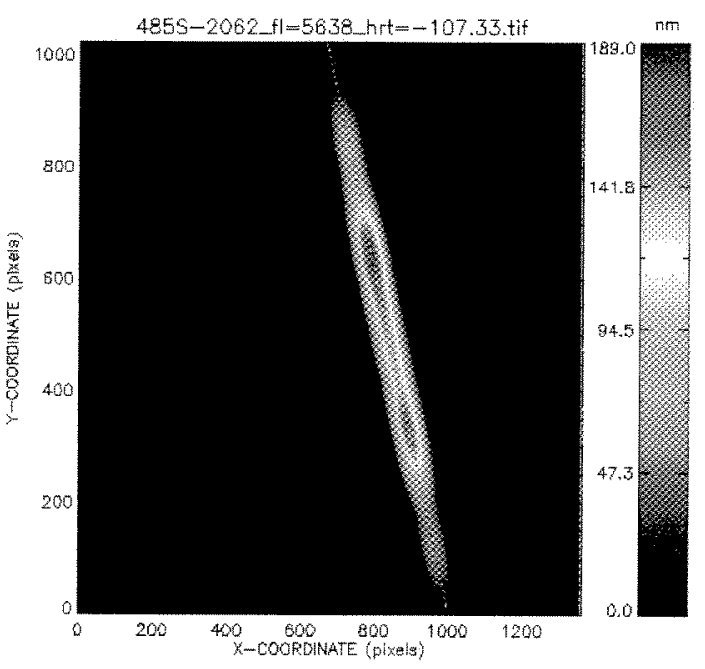

Figure 9 - Analyzed single image from Hartmann test

Once this line has been found, the points along the line are analyzed to compare the brightness of each pixel. The light intensity as a function of focal plane coordinate is shown in Figure 10. The centroid of the area under this curve is calculated to determine image's centroid. This centroid represents where the center of the hourglass is for that specific image. By comparing the centroids of all of the images, the error rating of the mirror can be determined as shown in Figure 8. The result obtained in Figure 9 closely resembles Figure 12 showing that the diffraction effect does indeed play a large role when using visible light. For this reason, the final test of the mirror alignment is done using $\mathrm{x}$ rays in a vacuum chamber. $X$-rays have a much shorter wavelength, so the diffraction effect is much smaller.

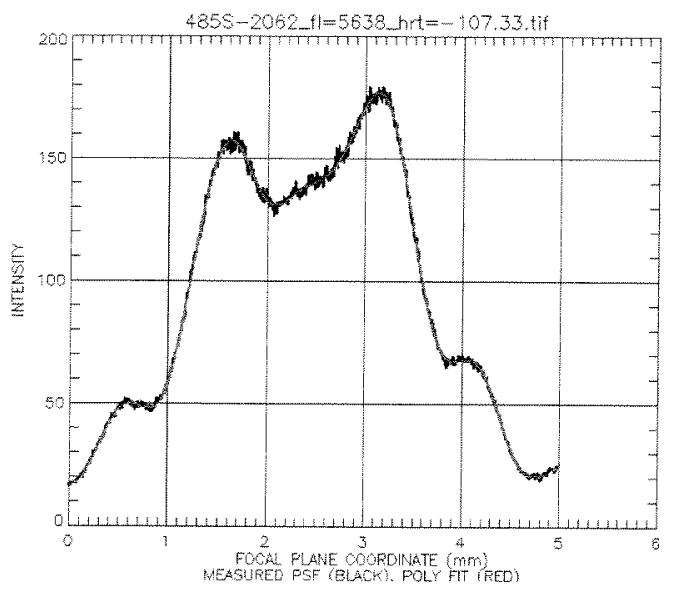

Figure 10 - Light intensity curve along sliver of light 


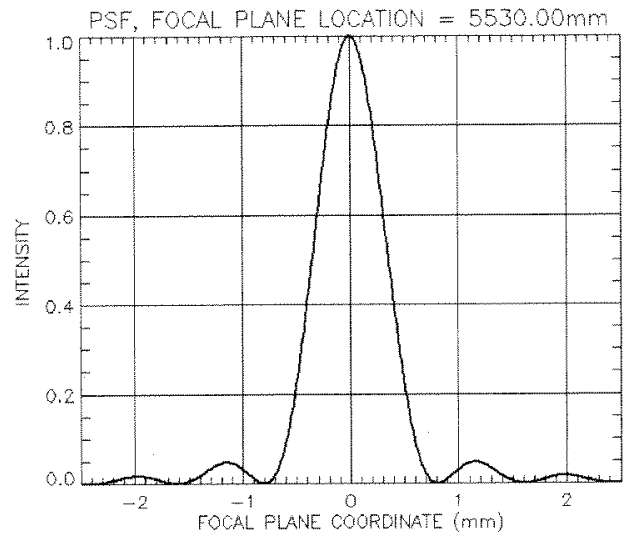

Figure 11 - Theoretical light intensity curve without diffraction

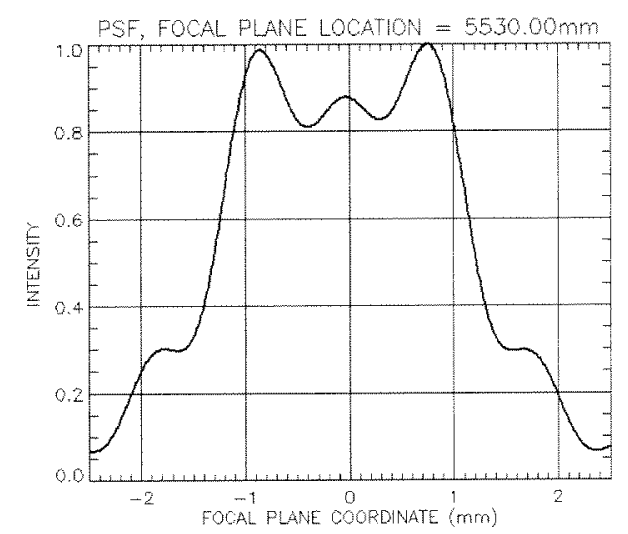

\section{Figure 12 - Theoretical light intensity curve with diffraction}

A repeatability test was performed to check the equipment by performing ten consecutive Hartmann tests at the same mirror alignment position and focal distance within a span of 30 minutes to minimize environmental changes. It was found that each individual centroid was repeatable to \pm 0.6 arcsec, and \pm 0.17 arcsec for the overall RMS mirror rating value.

\section{PERMANENT BONDING}

Once a mirror segment has been properly aligned, it is permanently bonded into a mock-up of the spacecraft module. For testing purposes, a Mirror Housing Simulator (MHS) is being used to emulate bond locations similar to the final module design. The MHS is capable of supporting three mirror pairs of different radii. The MHS is constructed of a Ti-15Mo alloy to closely match the coefficient of thermal expansion (CTE) of the D263 glass mirror segments.

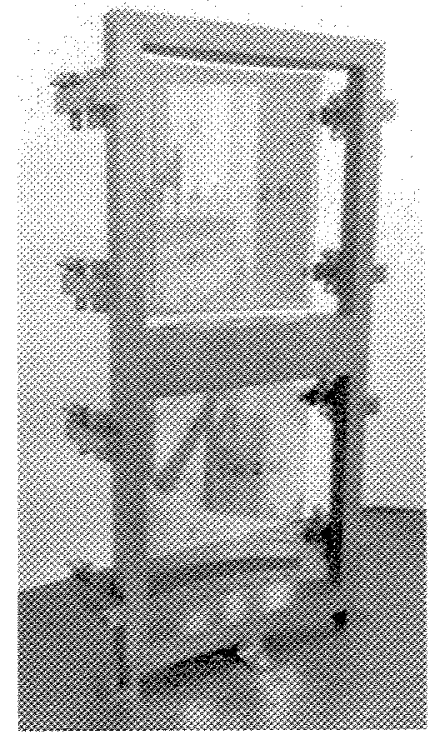

\section{Figure 13 - Mirror Housing Simulator (MHS)}

There are twelve rails, six on each side to hold the primary and secondary mirrors. For current testing purposes only the rails at the four corners of each mirror are being used as shown in Figure 13. Small flat tabs slide along the rails into position behind the mirror segment as shown in Figure 14. Once in position, the tabs are glued to the rail using Optocast 3408 UV cure epoxy.

The epoxy injection process has been automated by using a robotic arm to rapidly position the syringe of epoxy behind each tab. Optocast 3408 UV cure epoxy is injected to bond the mirror to the tab. Once the mirror has been bonded to all four tabs, the temporary bonds are broken by twisting the pins, and the strongback is removed. It has been demonstrated that breaking the temporary bonds does not damage the mirror.

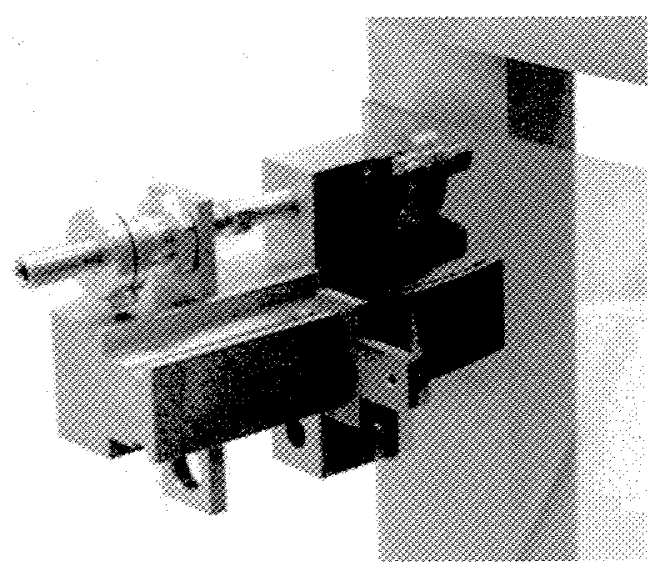

Figure 14 - MHS rail with tab and mirror

A detailed study is underway to determine a method to bond the mirror to the tab while imposing less than 0.3 microns of displacement. This is the perceived allotment of shift in mirror position that would be allowed under the current 
error budget scenario for preserving the shape of the mirror for acceptable optical quality.

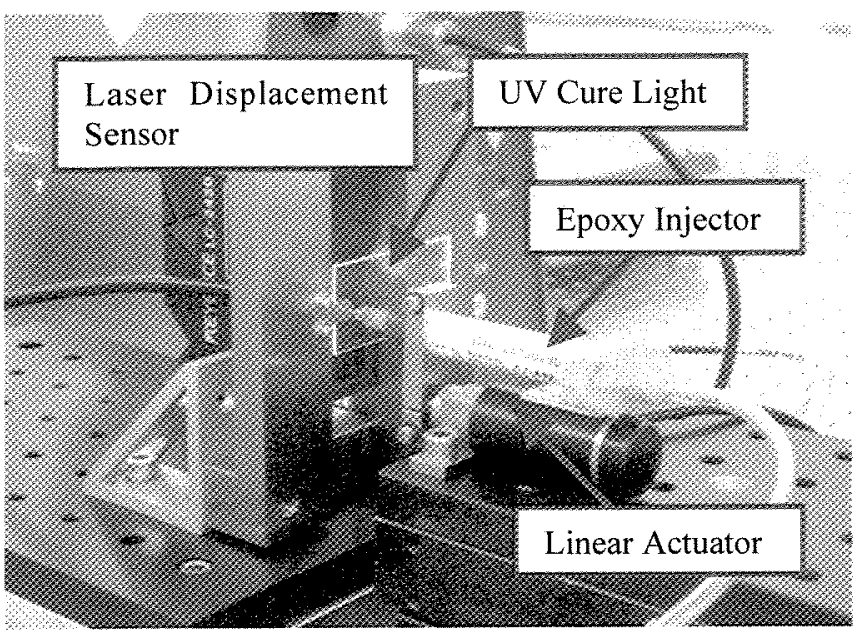

Figure 15 - Epoxy injector mounted to linear actuator

To achieve the submicron mirror displacement due to applying epoxy a zero-displacement bond method is being developed. A small high resolution linear actuator with a resolution of $30 \mathrm{~nm}$ is used to move the syringe. The actuator is wired into a closed loop system utilizing a laser displacement sensor with a resolution of $0.01 \mu \mathrm{m}$. The actuator oscillates the syringe tip in and out of the tab to move the mirror using the viscous forces from the liquid epoxy. The syringe is oscillated until the mirror has reached the desired offset position. This offset is determined by how much epoxy shrinkage will occur during the cure using the UV light. The epoxy is then cured, bringing the final displacement to zero. The setup for this process is depicted in Figure 15.

\section{Conclusions}

The mission requirements for IXO of large effective area and high angular resolution do not leave much room for error in the alignment and mounting of thin mirror segments. However, this has driven the design of new hardware and procedures to accommodate these challenges. The automation of the Hartmann test and on-site dataanalysis has made it possible to develop an iterative process to optimize the alignment of the mirror. In addition, the automation of the bonding process has led to advances in deformation control to the sub-micron level. Given the strict error budget allowed in the alignment and bonding of a mirror segment to its permanent housing, these advances are significant. Because of the modular design of the FMA this work should apply directly to the other segments to help make this mission a reality.

Future work includes bonding a secondary mirror with less than one arc second change in Hartmann test results before and after bonding. The same procedure will be repeated for a primary mirror, and then for a primary/secondary mirror pair. Once this is achieved, the co-alignment of nested mirror pairs will be tested.

\section{REFERENCES}

[1] J. Bookbinder, R. Smith, A. Hornschemeier, et al, "The Constellation-X Observatory" Proc. of SPIE Vol. 7011, $701102,2008$.

[2] R. McClelland and D. Robinson, "Design Concept for the International X-Ray Observatory Flight Mirror Assembly," 2009 IEEE Aerospace Conference Proceedings, March 7-14, 2009.

[3] W. Podgorski, D. Caldwell, M. Freeman, et al., "A Mounting and Alignment Approach for Constellation-X Mirror Segments," Proc. of SPIE, 7011, 701112, 2008.

[4] M. Beijersbergen, S. Kraft, R. Gunther, et al, "Silicon Pore Optics: Novel Lightweight High-Resolution X-Ray Optics Developed for XEUS," Proc. of SPIE, Vol. 5488, $868,2004$.

[5] W. Zhang, et al., "IXO Mirror Technology Development" Proc. of SPIE, Vol. 7437, 2009.

[6] K-W. Chan, W. Zhang, T. Saha, et al., "An Approach for Alignment, Mounting and Integration of IXO Mirror Segments," Proc. of SPIE, Vol. 7437, 2009.

\section{BIOGRAPHY}

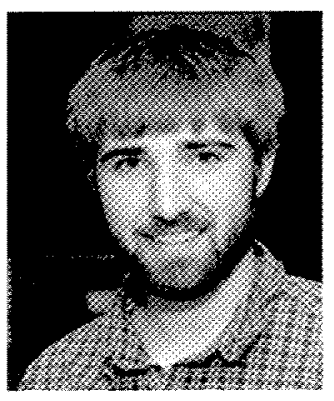

Tyler Evans is a Mechanical Systems Engineer at SGT Inc. currently working on the alignment and bonding of mirror segments for IXO at the NASA Goddard Space Flight Center. He received a B.S. in Mechanical Engineering from Purdue University.

Kai-Wing Chan is a Research Scientist at Universities Space Research Association currently working on development of the $x$-ray mirror assembly for $I X O$ at the NASA Goddard Space Flight Center. His previous experience includes work on the x-ray telescopes for the Japan-U.S. mission Suzaku (formerly Astro-E2). Prior to Suzaku, he was a member of the mirror team which developed the X-Ray Telescopes for the original Astro-E and inFOCuS. He received a B.S. in Physics from the Chinese University of Hong Kong, and a M.S. and Ph.D. in Physics from the University of California, San Diego.

\section{ACKNOWLEDGEMENTS}

Many people have contributed to the IXO mirror technology development. Those that have helped include: Glenn Byron, 
Kai-Wing Chan, Tyler Evans, Charles Fleetwood, Melinda Hong, Jacob Larimore, James Mazzarella, Ryan McClelland, Lawrence Olsen, David Robinson, Don Righter, Timo Saha, Kaitlyn Yoha, and William Zhang. 